\title{
TCG: A Transitive Closure Graph-Based Representation for General Floorplans
}

\author{
Jai-Ming Lin and Yao-Wen Chang, Member, IEEE
}

\begin{abstract}
In this paper, we introduce the concept of the $\mathbf{P}^{*}$-admissible representation and propose a $\mathbf{P} *$-admissible, transitive closure graph-based representation for general floorplans, called $T C G$, and show its superior properties. TCG combines the advantages of popular representations such as sequence pair, BSG, and B*-tree. Like sequence pair and BSG, but unlike O-tree, $B *$-tree, and CBL, TCG is $P^{*}$-admissible. Like $B^{*}$-tree, but unlike sequence pair, BSG, O-tree, and CBL, TCG does not need to construct additional constraint graphs for the cost evaluation during packing, implying faster runtime. Further, TCG supports incremental update during operations and keeps the information of boundary modules as well as the shapes and the relative positions of modules in the representation. More importantly, the geometric relation among modules is transparent not only to the TCG representation but also to its operations, facilitating the convergence to a desired solution. All these properties make TCG an effective and flexible representation for handling the general floorplan/placement design problems with various constraints. Experimental results show the promise of TCG.
\end{abstract}

Keywords: Floorplanning, Layout, Physical Design, Transitive Closure Graph

\section{INTRODUCTION}

As technology advances, circuit sizes and design complexity in modern VLSI design are increasing rapidly. To handle the design complexity, hierarchical design and reuse of IP modules become popular, which makes floorplanning/placement much more important than ever. The major objective of floorplanning/placement is to allocate the modules of a circuit into a chip to optimize some design metric such as area and timing. The realization of floorplanning/placement relies on a representation which describes geometric relations among modules. The representation has a great impact on the feasibility and complexity of floorplan designs. Thus, it is of particular significance to develop an efficient, effective, and flexible representation for floorplan/placement designs.

\section{A. Previous Work}

There exist a few floorplan representations in the literature, e.g., [1], [2], [3], [7], [8], [9], [10], [11], [12], [13], [17], [18]. We shall first review these representations and the types of floorplans that they can represent. A slicing floorplan is one of the simplest type of floorplans. A slicing structure can be obtained by recursively cutting rectangles horizontally or vertically into smaller rectangles; it is a non-slicing structure, otherwise. Otten first proposed a binary-tree representation for slicing floorplans [11]. Wong and Liu later in [18] presented a normal-

Manuscript received Sep 7, 2002; revised Feb 28, 2003. This paper was recommended by Editor-in-Chief Nagarajan Ranganathan.

This work was supported in part by the National Science Council of Taiwan ROC by Grant No. NSC-89-2215-E-009-117.

Jai-Ming Lin is with Realtek Semiconductor Corp. in Hsinchu Science-Based Industrial Park, Hsinchu 300, Taiwan. E-mail: gis87808@ cis.nctu.edu.tw.

Yao-Wen Chang is with the Department of Electrical Engineering \& the Graduate Institute of Electronics Engineering, National Taiwan University, Taipei 106, Taiwan. E-mail: ywchang@cc.ee.ntu.edu.tw. ized Polish expression (NPE for short) to represent a slicing floorplan. The slicing structure has several advantages such as smaller solution space, implying faster runtime for floorplan design. However, most of real designs are non-slicing. Researchers in [12] and [17] attempted to extend the tree representation to the non-slicing floorplans with special topologies, e.g., the wheel structure.

For the non-slicing floorplan structure, there exist several well-known "old" graph-based representations. Ohtsuki in [9] used a pair of horizontal and vertical directed acyclic graphs, named polar graphs, to represent a topological placement. Other representations such as adjacency graphs, and channel intersection graph are also widely used [14]. Recently, Onodera et al. in [10] used a branch-and-bound method to exhaustively search an optimal solution for module placement. Since the method is quite time-consuming, the size of tractable modules is limited.

The non-slicing floorplan representations have attracted much attention in the literature recently, e.g., sequence pair [7], bounded sliceline grid [8], O-tree [2], $B^{*}$-tree [1], and corner block list [3]. Murata et al. in [7] used two sequences $\left(\Gamma_{+}, \Gamma_{-}\right)$ of module names, called sequence pair (SP for short), to represent the geometric relations among modules. They defined the $P$-admissible solution space, which satisfies the following four requirements [7]:

(1) the solution space is finite,

(2) every solution is feasible,

(3) packing and cost evaluation can be performed in polynomial time, and

(4) the best evaluated packing in the space corresponds to an optimal placement.

(By this definition, the slicing tree is not a $\mathrm{P}$-admissible representation since many optimal floorplans are non-slicing.) SP is P-admissible and is flexible for general floorplan/placement design; however, it is harder to handle the floorplan/placement problems with position constraints, e.g., boundary modules, preplaced modules, range constraints, etc. Further, two constraint graphs need to be constructed for cost evaluation for each perturbation, consuming a significantly larger running time. Tang and Wong [16] recently presented an efficient packing scheme, called FAST-SP, to evaluate the cost of a sequence pair by computing its common subsequence. Nakatake et al. in [8] proposed a flexible bounded sliceline grid representation, called BSG. BSG is also P-admissible. However, BSG itself has many redundancies since there could be multiple representations corresponding to one packing, implying a larger solution space and thus longer search time to find an optimal solution.

For tree-based methods, Guo et al. in [2] proposed the Otree representation for a left and bottom compacted placement. 
(A similar idea to the O-tree was independently developed by Takahashi in [15].) In an O-tree, a node denotes a module and an edge denotes the horizontal adjacency relation of two modules. The O-tree can pack modules in linear time, but it needs to perform a sequence of operations to make a placement compacted to the left and the bottom to obtain a feasible O-tree. Such an operation may require an overall quadratic time complexity since it may need a linear number of transformations between O-trees and their corresponding placements (constraint graphs), and each of the transformation takes linear time. Further, the transformation may result in a mismatch between the original representation and its placement, harming the solution structure. Chang et al. recently in [1] presented a binary treebased representation for a left and bottom compacted placement, called $\mathrm{B}^{*}$-tree, and showed its superior properties for operations. In a $\mathrm{B}^{*}$-tree, a node denotes a module, the left child of a node represents the lowest adjacent module on the right, and the right child represents the first module above and with the same $x$ coordinate. Similar to O-tree, the representation could be changed after packing since the space intended for placing a module may be occupied by a previously placed module. Therefore, given an $\mathrm{O}$-tree or a $\mathrm{B}^{*}$-tree, it may not be feasible to find a placement corresponding to its original representation. Since the treebased representations can represent only compacted floorplans, they induce smaller solution spaces and have lower complexity for a single packing operation than SP and BSG. However, they might lead to only suboptimal solutions for some cost metric (such as wirelength) since it is very likely that the optimum ones occur when modules are not compacted. Fig 1(a) shows an uncompacted placement that cannot be represented by any $\mathrm{O}-$ tree (since module $b$ is not compacted to module $a$ ). Therefore, the O-tree representation will fail to find the optimum solution for wirelength optimization if modules $b$ and $c$ are strongly connected.

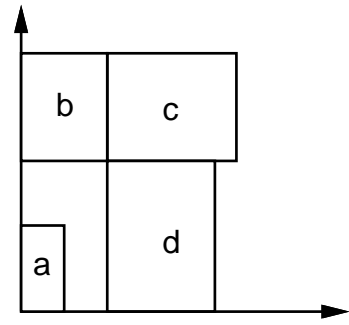

(a)

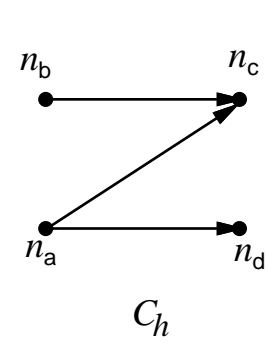

(b)

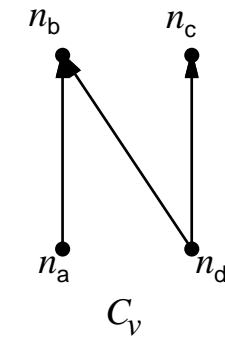

Fig. 1. (a) An uncompacted placement that cannot be represented by any O-tree. (b) The TCG representation for the placement shown in (a).

Recently, Hong et al. in [3] proposed a corner block list (CBL) representation for mosaic floorplans. A CBL consists of a 3tuple $(S, L, T)$, where $S$ is a sequence of corner modules, $L$ is a list of module orientations ( 0 for a vertical $T$-junction at the corner, and 1 for a horizontal one), and $T$ is a list of $T$-junction information. In a mosaic floorplan, each region must contain exactly one module. Obviously, such restriction makes its solution space smaller than SP and BSG. However, CBL is not Padmissible since it cannot guarantee a feasible solution in each perturbation, and many infeasible solutions may be generated before a feasible solution is found.

\section{B. Our Contribution}

We propose in this paper a transitive closure graph-based representation for general non-slicing floorplans, named $T C G$, and show its superior properties. TCG uses a horizontal and a vertical transitive closure graphs, $C_{h}$ and $C_{v}$, to describe the horizontal and vertical relations for each pair of modules. In the $C_{h}$ $\left(C_{v}\right)$ of Fig 1(b), for example, an edge from module $x$ to module $y$ represents that $x$ is left to (below) $y$. (We will give a formal definition on the graphs later.) TCG is the first representation that can perturb on (non-tree) graphs directly and guarantee a feasible solution in each perturbation.

To differentiate the properties of TCG from other existing representations, we extend in this paper the concept of the $\mathrm{P}$ admissible representation to that of the $P^{*}$-admissible one by adding the following condition:

(5) the geometric relation between each pair of modules is defined in the representation.

The fifth condition facilitates the handling of the floorplan/placement design problems with additional requirements such as module sizing and position constraints (e.g., boundary constraints, symmetry constraints, etc). The representation after packing corresponds to the original one if it satisfies the condition. It leads to a better solution (neighborhood) structure, facilitating the search for an optimum solution. The $\mathrm{P}^{*}$-admissible representation corresponds to a general topological modeling of modules, and thus contains a complete structure for searching for an optimum floorplan/placement solution. For example, for the placement of Fig 1(a) that cannot be represented by any Otree, it can easily be represented by the TCG (a P*-admissible representation) shown in Fig 1(b). Due to the elegant properties, it is desirable to develop an effective and flexible $\mathrm{P}^{*}$-admissible representation.

Among the existing popular representations, SP, BSG, and TCG are $\mathrm{P}^{*}$-admissible while slicing trees, NPE, O-tree, $\mathrm{B} *_{-}$ tree, and CBL are not. Slicing trees, NPE, and CBL are not $\mathrm{P}$-admissible and thus non-P*-admissible. The tree-based representations violates both the fifth condition of the $\mathrm{P}^{*}$ admissibility. The insufficiency (due to the oversimplified representations) incurs the following drawbacks:

- Some geometric relations between modules cannot be obtained from the $\mathrm{O}$-tree and the $\mathrm{B}^{*}$-tree representations directly, making $\mathrm{O}$-trees and $\mathrm{B} *$-trees harder to handle the flooprlan design problems with the aforementioned additional requirements. Fig 2(a) shows a compacted placement with five modules $a, b, c$, $d$, and $e$ whose widths and heights are $(6,4),(4,6),(7,4),(6,3)$ and $(3,2)$, respectively. Fig 2(b) and (c) show the O-tree and the $\mathrm{B} *$-tree corresponding to the placement of Fig 2(a), respectively. As illustrated in the figures, we cannot derive any geometric relation between two modules from the O-tree and $\mathrm{B} *$-tree unless the two corresponding nodes are siblings or on the same path. For example, even though module $b$ is adjacent to module $d$ in the placement, we cannot derive any geometric relation from the representations directly until packing. Further, the geometric relation between two modules for the same $\mathrm{O}$-tree or $\mathrm{B}$ *-tree may change if the dimensions of modules are changed. For example, if the dimension of module $b$ is changed to $(1,6)$, module $d$ is 
right to module $b$ after packing as shown in Fig 2(d), instead of being above $b$ as in Fig 2(a) (for the same O-tree shown in Fig 2(b)). The mismatch would inevitably complicate the floorplan design process.

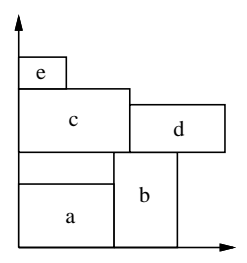

(a)

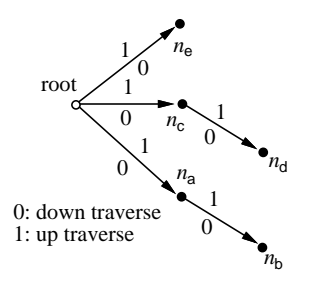

(b)

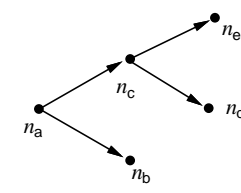

(c) (d)

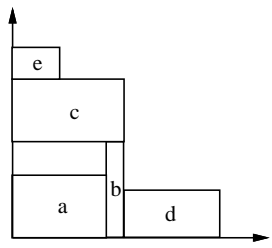

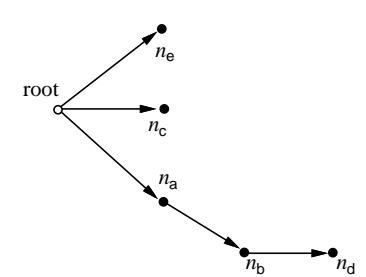

(e)
Fig. 2. (a) A placement. (b) The corresponding O-tree. (c) The corresponding $\mathrm{B}^{*}$-tree. (d) The placement after packing if the dimension of module $b$ is changed to $(1,6)$. (e) The O-tree derived from the placement of (d).

Also, for module sizing, it is better to keep the geometric relation between each pair of modules in the representation to prevent a re-sized module from overlapping with other modules. Besides, it is harder to handle boundary and symmetry modules with the tree-based representations. For the O-tree shown in Fig 2(b), $n_{b}$ seems to denote a right boundary module because there exists no node on its right side. However, module $b$ may not be a right boundary module in the final placement as shown in Fig 2(d). To deal with symmetry constraints, several pairs of modules have to be placed symmetrically with respect to a common axis, and the $x$ or $y$ coordinates of the modules in each pair must be the same. It is desirable to keep the geometric relation between modules in the representation to facilitate the floorplan/placement design with symmetry modules.

- Due to their compaction operations, the O-tree or the B*-tree after packing may not correspond to the original one, which may harm the solution (neighborhood) structure and thus also the convergence to an optimum solution. After packing, for example, the initial O-tree of Fig 2(b) results in the placement of Fig 2(d) which corresponds to a different O-tree shown in Fig 2(e).

Despite of their smaller solution spaces and cheaper single packing complexity, the aforementioned drawbacks make non-P*admissible representations less flexible and effective in handling practical floorplan/placement design problems which need to consider various requirements.

In contrast, TCG combines the advantages of SP, BSG, and $\mathrm{B} *$-tree. Like SP and BSG, but unlike O-tree, B*-tree, and CBL, TCG satisfies the five conditions of $\mathrm{P}^{*}$-admissibility:

(1) its solution space is $(m !)^{2}$ and thus finite, where $m$ is the number of modules,

(2) every solution is feasible (note that the CBL representation does not guarantee this property),
(3) packing and cost evaluation can be performed in $O\left(\mathrm{~m}^{2}\right)$ time,

(4) the best evaluated packing in the solution space corresponds to an optimum placement,

(5) the geometric relation between each pair of modules is defined in the TCG representation.

The solution space is the same as SP but the memory usage is smaller since we do not need to maintain a sequence pair. Like B*-tree, but unlike SP, BSG, O-tree, and CBL, TCG does not need to construct additional constraint graphs for the cost evaluation during packing, implying faster running time. Further, TCG supports incremental update during operations, and keeps the information of boundary modules as well as the shapes and the relative positions of modules in the representation. More importantly, the geometric relation among modules is transparent not only to the TCG representation but also to its operations (i.e., the effect of an operation on the change of the geometric relation is known before packing), facilitating faster convergence to a desired solution and placement with position constraints. For example, as illustrated in Fig 1, the nodes with zero indegree (out-degree) in the horizontal constraint graph $C_{h}$ correspond to the left (right) boundary modules in the placement, and the nodes with zero in-degree (out-degree) in the vertical constraint graph $C_{v}$ correspond to the bottom (top) boundary modules. The transparency of the geometric relation among modules distinguishes TCG from other representations in handling placement with position constraints. All these properties make TCG an effective and flexible representation for handling the general floorplan/placement design problems with various requirements. Experimental results show the promise of TCG. For area optimization, TCG achieved average improvements of $2.22 \%$, $2.04 \%, 1.18 \%$, and $3.54 \%$, compared to O-tree, enhanced Otree, $\mathrm{B}^{*}$-tree, and $\mathrm{CBL}$, respectively. Optimizing wirelength, TCG obtained respective average improvements of $3.56 \%$ and $3.18 \%$, compared to O-tree and enhanced O-tree. (Note that $\mathrm{B} *_{-}$ tree and CBL do not report the results for optimizing wirelength alone.) The runtime requirements of TCG are much smaller than $\mathrm{O}$-tree and $\mathrm{B} *$-tree, and are comparable to enhanced $\mathrm{O}$-tree.

The remainder of this paper is organized as follows. Section II formulates the floorplan/placement design problem. Section III presents the procedures to derive a TCG from a placement and construct a placement from a TCG. Section IV introduces the operations to perturb a TCG. Experimental results are reported in Section V. Finally, we conclude our work and discuss future research directions in Section VI.

\section{Problem Definition}

Let $B=\left\{b_{1}, b_{2}, \ldots, b_{m}\right\}$ be a set of $m$ rectangular modules whose width, height, and area are denoted by $W_{i}, H_{i}$, and $A_{i}$, $1 \leq i \leq m$. Each module is free to rotate. Let $\left(x_{i}, y_{i}\right)$ denote the coordinate of the bottom-left corner of rectangle $b_{i}, 1 \leq$ $i \leq m$, on a chip. A placement $\mathcal{P}$ is an assignment of $\left(x_{i}, y_{i}\right)$ for each $b_{i}, 1 \leq i \leq m$, such that no two modules overlap. The goal of floorplanning/placement is to optimize a predefined cost metric such as a combination of the area (i.e., the minimum bounding rectangle of $\mathcal{P}$ ) and wirelength (i.e., the summation of half bounding box of interconnections) induced by a placement. 


\section{Transitive Closure Graph (TCG)}

The transitive closure of a directed acyclic graph $G$ is defined as the graph $G^{\prime}=\left(V, E^{\prime}\right)$, where $E^{\prime}=\left\{\left(n_{i}, n_{j}\right)\right.$ : there is a path from node $n_{i}$ to node $n_{j}$ in $\left.G\right\}$. The Transitive Closure Graph (TCG) representation describes the geometric relations among modules based on two graphs, namely a horizontal transitive closure graph $C_{h}$ and a vertical transitive closure graph $C_{v}$. In this section, we first introduce the procedure for constructing $C_{h}$ and $C_{v}$ from a placement. Then, we describe how to pack modules from TCG. In the last subsection, we discuss the properties and the solution space of TCG.

\section{A. From a placement to its TCG}

For two non-overlapped modules $b_{i}$ and $b_{j}, b_{i}$ is said to be horizontally (vertically) related to $b_{j}$, denoted by $b_{i} \vdash b_{j}\left(b_{i} \perp\right.$ $b_{j}$ ), if $b_{i}$ is on the left (bottom) side of $b_{j}$ and their projections on the $y(x)$ axis overlap. (Note that two modules cannot have both horizontal and vertical relations unless they overlap.) For two non-overlapped modules $b_{i}$ and $b_{j}, b_{i}$ is said to be diagonally related to $b_{j}$ if $b_{i}$ is on the left side of $b_{j}$ and their projections on the $x$ and the $y$ axes do not overlap. In a placement, every two modules must bear one of the three relations: horizontal relation, vertical relation, and diagonal relation. To simplify the operations on geometric relations, we treat a diagonal relation for modules $b_{i}$ and $b_{j}$ as a horizontal one, unless there exists a chain of vertical relations from $b_{i}\left(b_{j}\right)$, followed by the modules enclosed with the rectangle defined by the two closest corners of $b_{i}$ and $b_{j}$, and finally to $b_{j}\left(b_{i}\right)$, for which we make $b_{i} \perp b_{j}$ $\left(b_{j} \perp b_{i}\right)$.

Fig 3(a) shows a placement with five modules $a, b, c, d$, and $e$ whose widths and heights are $(6,4),(4,6),(7,4),(6,3)$ and $(3,2)$, respectively. In Fig 3(a), $a \vdash b, a \perp c$, and module $e$ is diagonally related to module $b$. There exists a chain of vertical relations formed by modules $e, c$, and $b$ between the two modules $e$ and $b$ (i.e., $b \perp c$ and $c \perp e$ ). Therefore, we make $b \perp e$. Also, module $e$ is diagonally related to module $d$. However, there dose not exist a chain of vertical relations between modules $e$ and $d$, and thus we make $e \vdash d$.

TCG can be derived from a placement as follows. For each module $b_{i}$ in a placement, we introduce a node $n_{i}$ with the weight being the width (height) in $C_{h}\left(C_{v}\right)$. If $b_{i} \vdash b_{j}$, we construct a directed edge from node $n_{i}$ to node $n_{j}$ (denoted by $\left.\left(n_{i}, n_{j}\right)\right)$ in $C_{h}$. Similarly, we construct a directed edge $\left(n_{i}, n_{j}\right)$ in $C_{v}$ if $b_{i} \perp b_{j}$. Given a placement with $m$ modules, we need to perform the above process $m(m-1) / 2$ times to capture all the geometric relations among modules (i.e., $C_{h}$ and $C_{v}$ have $m(m-1) / 2$ edges in total).

As shown in Fig 3(b), for each module $b_{i}, i \in\{a, b, c, d$, $e\}$, we introduce a node $n_{i}$ in $C_{h}$ and also in $C_{v}$. For each node $n_{i}$ in $C_{h}\left(C_{v}\right), i \in\{a, b, c, d, e\}$, we associate the node with a weight equal to the width (height) of the corresponding module $b_{i}$. Since $b_{a} \vdash b_{b}$, we construct a directed edge ( $n_{a}$, $\left.n_{b}\right)$ in $C_{h}$. Similarly, we construct a directed edge $\left(n_{a}, n_{c}\right)$ in $C_{v}$ since $b_{a} \perp b_{c}$. This process is repeated until all geometric relations among modules are defined. As shown in Fig 3(b), each transitive closure graph has five nodes, and there are totally 10 edges in $C_{h}$ and $C_{v}$ (four in $C_{h}$ and six in $C_{v}$ ). From the
TCG representation shown in Fig 3(b), we know that module $e$ is above module $b$ because there exists a directed edge $\left(n_{b}\right.$, $n_{e}$ ) in $C_{v}$; notice that this relation cannot be directly derived from the O-tree and $\mathrm{B}^{*}$-tree shown in Fig 2. Further, we also know directly from the TCG that module $d$ is right to module $a$ while the relationship is not known to O-tree and $\mathrm{B} *$-tree until modules are placed. For boundary information, we know that modules $a, c$ and $e$ ( $b$ and $d$ ) are on the left (right) boundary since the in-degrees (out-degrees) of $n_{a}, n_{c}$ and $n_{e}\left(n_{b}\right.$ and $\left.n_{d}\right)$ are zero in $C_{h}$. Similarly, it is easy to know from $C_{v}$ that modules $a$ and $b(e$ and $d)$ are on the bottom (top) boundary. Therefore, the floorplan/placement design with boundary constraints can be handled easily by checking the degree of a node during each perturbation.

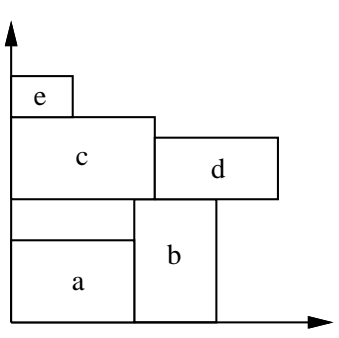

(a)

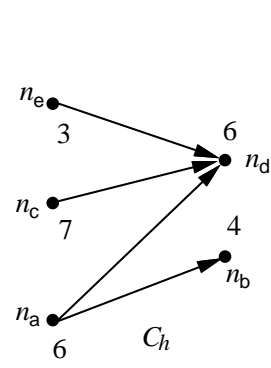

(b)

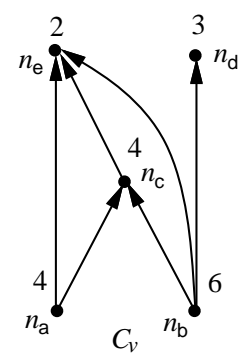

Fig. 3. (a) A placement in a chip. (b) TCG.

\section{B. From a TCG to its placement}

We have introduced how to derive a TCG from its placement in the previous section. We now present the packing method for a TCG.

Given a TCG, its corresponding placement can be obtained in $O\left(\mathrm{~m}^{2}\right)$ time by performing a well-known longest path algorithm [5] on the TCG, where $m$ is the number of modules. To facilitate the implementation of the longest path algorithm, we augment the given two closure graphs as follows. (Note that the TCG augmentation is performed only for packing. It will be clear later that such augmentation is not needed for other operations such as solution perturbation.) We introduce two special nodes with zero weights for each closure graph, the source $n_{s}$ and the sink $n_{t}$, and construct an edge from $n_{s}$ to each node with in-degree equal to zero, and also from each node with outdegree equal to zero to $n_{t}$. Fig 4 shows the augmented TCG for the TCG shown in Fig 3(b).

Let $L_{h}\left(n_{i}\right)\left(L_{v}\left(n_{i}\right)\right)$ be the length of the longest path from $n_{s}$ to $n_{i}$ in the augmented $C_{h}\left(C_{v}\right) . L_{h}\left(n_{i}\right)\left(L_{v}\left(n_{i}\right)\right)$ can be determined by performing the single source longest path algorithm on the augmented $C_{h}\left(C_{v}\right)$ in $O\left(m^{2}\right)$ time, where $m$ is number of modules. The coordinate $\left(x_{i}, y_{i}\right)$ of a module $b_{i}$ is given by $\left(L_{h}\left(n_{i}\right), L_{v}\left(n_{i}\right)\right)$. Since the respective width and height of the placement for the given TCG are $L_{h}\left(n_{t}\right)$ and $L_{v}\left(n_{t}\right)$, the area of the placement is given by $L_{h}\left(n_{t}\right) L_{v}\left(n_{t}\right)$.

\section{Properties of TCG}

Property 1: (TCG Feasibility Conditions) A feasible TCG has the following three properties:

(1) $C_{h}$ and $C_{v}$ are acyclic. 


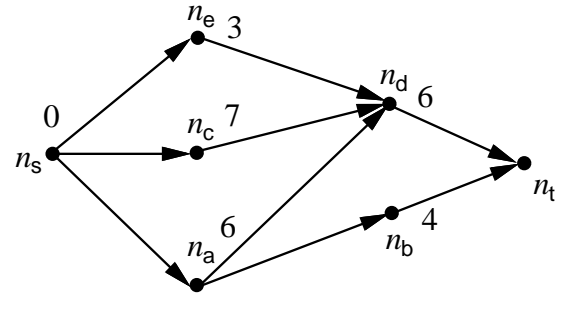

(a)

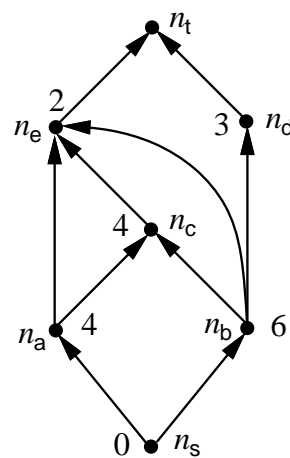

(b)
Fig. 4. Augmented TCG. (a) Augmented $C_{h}$. (b) Augmented $C_{v}$.

(2) Each pair of nodes must be connected by exactly one edge either in $C_{h}$ or in $C_{v}$.

(3) The transitive closure of $C_{h}\left(C_{v}\right)$ is equal to $C_{h}\left(C_{v}\right)$ itself. Proof:

(1) For each pair of nodes, we construct a directed edge according to the geometrical relation of two modules. Since a module cannot be both left and right (below and above) to another module in a placement, the resulting graphs $C_{h}$ and $C_{v}$ must be acyclic.

(2) Given a placement with $m$ modules, as mentioned earlier, we construct $m(m-1) / 2$ edges to capture all geometric relations among modules. Since there are also $m(m-1) / 2$ pairs of nodes and no multiple edges are allowed, each pair of nodes would be connected by exactly one edge either in $C_{h}$ or in $C_{v}$. (3) To prove Property 3, we claim that $b_{i} \vdash b_{k}\left(b_{i} \perp b_{k}\right)$ if $b_{i} \vdash b_{j}$ and $b_{j} \vdash b_{k}\left(b_{i} \perp b_{j}\right.$ and $\left.b_{j} \perp b_{k}\right)$. Suppose $b_{i} \vdash b_{j}$ and $b_{j} \vdash b_{k}$, but we make $b_{i} \perp b_{k}$. This implies that all modules $b_{l}$ 's overlapped with the rectangle defined by the two closest corners of $b_{i}$ and $b_{k}$ have the geometric relations $b_{i} \perp b_{l}$ and $b_{l} \perp b_{k}$, which is a contradiction to our assumption that $b_{i} \vdash b_{j}$ and $b_{j} \vdash b_{k}$. Similarly, we claim that $b_{i} \perp b_{k}$ if $b_{i} \perp b_{j}$ and $b_{j} \perp b_{k}$.

Property 1 ensures that a module $b_{i}$ cannot be both left and right to (below and above) another module $b_{j}$ in a placement. Property 2 guarantees that no two modules overlap since each pair of modules have exactly one of the horizontal or vertical relation. Property 3 is used to eliminate redundant solutions. It guarantees that if there exists a path from $n_{i}$ to $n_{j}$ in one closure graph, the edge $\left(n_{i}, n_{j}\right)$ must also appear in the same closure graph. For example, there exist two edges $\left(n_{i}, n_{j}\right)$ and $\left(n_{j}, n_{k}\right)$ in $C_{h}$, which means that $b_{i} \vdash b_{j}$ and $b_{j} \vdash b_{k}$, and thus $b_{i} \vdash b_{k}$. If the edge $\left(n_{i}, n_{k}\right)$ appears in $C_{v}$ instead of in $C_{h}$, $b_{k}$ is not only left to $b_{i}$ but also above $b_{i}$. The resulting area of the corresponding placement must be larger than or equal to that when the edge $\left(n_{i}, n_{k}\right)$ appears in $C_{h}$. Fig 5 illustrates this phenomenon. In Fig 5(a), there exists a path from $n_{a}$ to $n_{e}$ in $c_{v}$, which consists of $\left(n_{a}, n_{c}\right)$ and $\left(n_{c}, n_{e}\right)$. Thus, the edge $\left(n_{a}, n_{e}\right)$ must also belong to $C_{v}$. If the edge $\left(n_{a}, n_{e}\right)$ appears in $C_{h}$ instead of in $C_{v}$ as shown in Fig 5(c), the resulting area of the placement must be larger than or equal to the configuration of Fig 5(a) and (b). Property 3 eliminates such a redundancy.

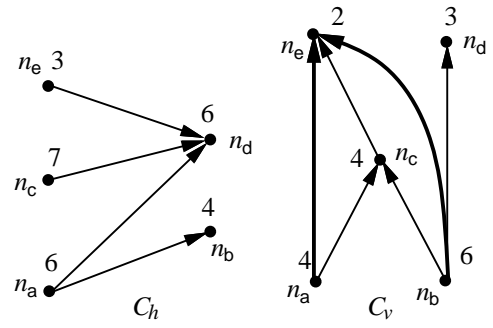

(a)

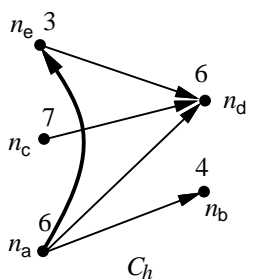

$C_{h}$

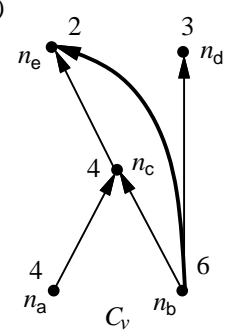

(c)

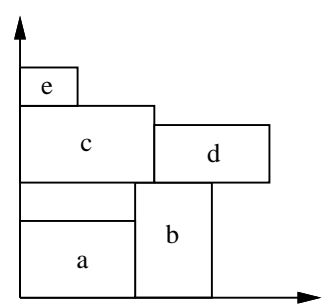

(b)

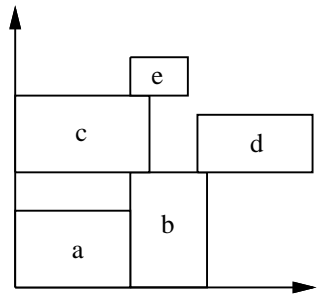

(d)
Fig. 5. Examples of the TCG feasibility. (a) A feasible TCG. (b) The placement corresponding to the TCG shown in (a). (c) A non-TCG. (d) The placement corresponding to the non-TCG shown in (c).

Based on the properties of TCG, we have the following theorems.

Theorem 1: There exists a unique placement corresponding to a TCG.

Proof: We first show that each TCG is feasible (i.e., there must exist a placement for each TCG), and then show the uniqueness of the placement.

Property 1 avoids that a module is both left and right to (or below and above) another module in the packing. Property 2 guarantees that no two modules overlap in the packing. Thus, Properties 1 and 2 guarantee that there exists a placement for each TCG. Given a TCG, the $x$ and $y$ coordinates of each module are determined by the respective longest paths in $C_{h}$ and $C_{v}$, which are well-defined values in the TCG. Therefore, the placement is unique.

Theorem 2: The size of the solution space for TCG is $(m !)^{2}$, where $m$ is the number of modules.

Proof: To show the size, we prove that there exists a oneto-one correspondence between a TCG $\left(C_{h}, C_{v}\right)$ and a sequence pair $\left(\Gamma_{+}, \Gamma_{-}\right)$. Since there are $(m !)^{2}$ such sequence pairs, the theorem thus follows.

Let the fan-in (fan-out) of a node $n_{i}$, denoted by $F_{i n}\left(n_{i}\right)$ $\left(F_{\text {out }}\left(n_{i}\right)\right)$, be the nodes $n_{j}$ 's with edges $\left(n_{j}, n_{i}\right)\left(\left(n_{i}, n_{j}\right)\right)$. Given a TCG, we can transform it into a sequence $\Gamma_{+}$by repeatedly extracting a node $n_{i}$ with $F_{i n}\left(n_{i}\right)=\emptyset$ in $C_{h}$ and $F_{\text {out }}\left(n_{i}\right)=\emptyset$ in $C_{v}$, and then deleting the edges $\left(n_{i}, n_{j}\right)$ 's ( $\left(n_{j}, n_{i}\right)$ 's) from $C_{h}\left(C_{v}\right)$ until no node is left in $C_{h}\left(C_{v}\right)$. Similarly, we can transform a TCG into another sequence $\Gamma_{-}$by repeatedly extracting the node $n_{i}$ with $F_{i n}\left(n_{i}\right)=\emptyset$ both in $C_{v}$ and $C_{h}$, and then deleting the edges $\left(n_{i}, n_{j}\right)$ 's from both $C_{h}$ and $C_{v}$ until no node is left in $C_{h}$ and $C_{v}$. As the example shown in Fig 3(a), we have $\Gamma_{+}=<n_{e}, n_{c}, n_{a}, n_{d}, n_{b}>$ and $\Gamma_{-}=<n_{a}, n_{b}, n_{c}, n_{e}, n_{d}>$.

We claim that there exists a unique sequence pair $\left(\Gamma_{+}, \Gamma_{-}\right)$ corresponding to a TCG. Since the node $n_{i}$ with $F_{i n}\left(n_{i}\right)=\emptyset$ in 
$C_{h}$ and $F_{\text {out }}\left(n_{i}\right)=\emptyset\left(F_{\text {in }}\left(n_{i}\right)=\emptyset\right)$ in $C_{v}$ denotes the unique module on the left and the top (bottom) boundaries of a placement, such a unique node can be found in each iteration during the transformation. By removing the node $n_{i}$ and its incident edges, we obtain a new TCG with fewer nodes. It is obvious that there is also a unique module on the left-top (left-bottom) corner in the placement corresponding to the new TCG. Repeating this process, we can transform a TCG into a unique sequence pair $\left(\Gamma_{+}, \Gamma_{-}\right)$.

Given a sequence pair $\left(\Gamma_{+}, \Gamma_{-}\right)$, we can obtain a unique TCG $\left(C_{h}, C_{v}\right)$ from the two constraint graphs induced from the sequence pair $\left(\Gamma_{+}, \Gamma_{-}\right)$by removing the source, the sink, and their associated edges in the graphs. To claim that the two resulting constraint graphs form a TCG, we shall prove that they satisfy the three properties of TCG. We shall first review the constraint graph construction for a sequence pair defined in [7]. Module $b_{a}$ is left (right) to module $b_{b}$ (i.e, there exists an edge $\left(n_{a}, n_{b}\right)$ $\left(\left(n_{b}, n_{a}\right)\right)$ in the horizontal constrain graph) if $a$ is before (after) $b$ in both $\Gamma_{+}$and $\Gamma_{-}$. Module $b_{a}$ is below (above) module $b_{b}$ (i.e, there exists an edge $\left(n_{a}, n_{b}\right)\left(\left(n_{b}, n_{a}\right)\right)$ in the vertical constrain graph) if $b$ is before (after) $a$ in $\Gamma_{+}$, and $a$ is before (after) $b$ in $\Gamma_{-}$.

- Property 1: Suppose there exists a cycle $\left\langle n_{a}, n_{b}, \ldots, n_{a}\right\rangle$ in the horizontal constraint graph of a sequence pair. Then, the corresponding $\Gamma_{+}$and $\Gamma_{-}$must be both in the sequence $\ldots a \ldots b \ldots a \ldots$, contradicting to the fact that a module cannot appear twice in a sequence. Similarly, there does not exist any cycle in $C_{v}$.

- Property 2: For every pair of nodes $n_{a}$ and $n_{b}$, there exists a unique edge $\left(n_{a}, n_{b}\right)$ or $\left(n_{b}, n_{a}\right)$ in a horizontal or a vertical constraint graph, depending on the relative positions of $a$ and $b$ in $\Gamma_{+}$and $\Gamma_{-}$.

- Property 3: To show that the transitive closure of a horizontal (vertical) constraint graph is equal to itself, we shall prove that if there exists a path $\left\langle n_{i}, n_{j}, n_{k}>\right.$ in a constraint graph, the edge $\left(n_{i}, n_{k}\right)$ also exists in the graph. If there exists a path $<$ $n_{i}, n_{j}, n_{k}>$ in the horizontal (vertical) constraint graph of a sequence pair, the sequence pair must be in this form $(\ldots i \ldots$ $j \ldots k \ldots, \ldots i \ldots j \ldots k \ldots)((\ldots k \ldots j \ldots i \ldots, \ldots i \ldots$ $j \ldots k \ldots)$, which implies that the edge $\left(n_{i}, n_{k}\right)$ also exists in the same graph.

The theorem thus follows.

According to the above discussions, we conclude the following theorem.

Theorem 3: $\mathrm{TCG}$ is $\mathrm{P}^{*}$-admissible.

We summarize in TABLE I the properties of several recently published representations for non-slicing floorplans ${ }^{1}$.

\section{Floorplanning Algorithm}

We develop a simulated annealing based algorithm [4] using TCG for non-slicing floorplan design. Given an initial solution represented by a TCG, the algorithm perturbs the TCG to obtain a new TCG. To ensure the correctness of the new TCG, as described in the previous section, the new TCG must satisfy the

\footnotetext{
${ }^{1}$ In [3], the authors claim that the solution space of CBL is $O\left(m ! 2^{3 m} / m^{1.5}\right)$. However, in the 3-tuple $(S, L, T)$ of CBL, there should be $m$ ! combinations for $S, 2^{m-1}$ combinations for $L$, and $2^{2 m-3}$ combinations for $T$; there its solution space should be $O\left(m ! 2^{3 m}\right)$
}

aforementioned three feasibility properties. To identify feasible TCG for perturbation, we introduce the concept of transitive reduction edges of TCG in the following section.

\section{A. Transitive Reduction Edges}

An edge $\left(n_{i}, n_{j}\right)$ is said to be a reduction edge if there does not exist another path from $n_{i}$ to $n_{j}$, except the edge $\left(n_{i}, n_{j}\right)$ itself; otherwise, it is a closure edge. For example, in the $C_{v}$ of Fig 5(a), the edges $\left(n_{a}, n_{c}\right),\left(n_{b}, n_{c}\right),\left(n_{c}, n_{e}\right)$, and $\left(n_{b}, n_{d}\right)$ are reduction edges while $\left(n_{a}, n_{e}\right)$ and $\left(n_{b}, n_{e}\right)$ are closure ones since there exist respective paths $\left\langle n_{a}, n_{c}, n_{e}>\right.$ and $<n_{b}, n_{c}, n_{e}>$ from $n_{a}$ to $n_{e}$ and from $n_{b}$ to $n_{e}$.

Since TCG is formed by directed acyclic transitive closure graphs, given an arbitrary node $n_{i}$ in one transitive closure graph, there exists at least one reduction edge $\left(n_{i}, n_{j}\right)$, where $n_{j} \in F_{\text {out }}\left(n_{i}\right)$. Here, we define the fan-in (fan-out) of a node $n_{i}$, denoted by $F_{\text {in }}\left(n_{i}\right)\left(F_{\text {out }}\left(n_{i}\right)\right)$, as the nodes $n_{j}$ 's with edges $\left(n_{j}, n_{i}\right)\left(\left(n_{i}, n_{j}\right)\right)$. For nodes $n_{k}, n_{l} \in F_{\text {out }}\left(n_{i}\right)$, the edge $\left(n_{i}, n_{k}\right)$ cannot be a reduction edge if $n_{k} \in F_{\text {out }}\left(n_{l}\right)$. Hence, we remove those nodes in $F_{\text {out }}\left(n_{i}\right)$ that are fan-outs of others. The edges between $n_{i}$ and the remaining nodes in $F_{\text {out }}\left(n_{i}\right)$ are reduction edges. For the $C_{v}$ shown in Fig 5(a), $F_{\text {out }}\left(n_{a}\right)=\left\{n_{c}, n_{e}\right\}$. Since $n_{e}$ belongs to $F_{\text {out }}\left(n_{c}\right)$, edge $\left(n_{a}, n_{e}\right)$ is a closure edge while $\left(n_{a}, n_{c}\right)$ is a reduction one.

Lemma 1: Given an arbitrary node $n_{i}$ in one transitive closure graph, for nodes $n_{k}, n_{l} \in F_{\text {out }}\left(n_{i}\right)$, the edge $\left(n_{i}, n_{k}\right)$ cannot be a reduction edge if $n_{k} \in F_{\text {out }}\left(n_{l}\right)$.

Proof: For nodes $n_{k}, n_{l} \in F_{\text {out }}\left(n_{i}\right)$ and $n_{k} \in F_{\text {out }}\left(n_{l}\right)$, the edge $\left(n_{i}, n_{k}\right)$ cannot be a reduction edge because there exists at least a path $\left\langle n_{i}, n_{l}, n_{k}>\right.$ from $n_{i}$ to $n_{k}$ except the edge $\left(n_{i}, n_{k}\right)$.

Theorem 4: Given a node $n_{i}$ in $C_{h}$ or $C_{v}$, it takes $O\left(m^{2}\right)$ time to find a reduction edge $\left(n_{i}, n_{j}\right)$, where $m$ is the number of modules.

Proof: Given a node $n_{i}$, there exist at most $m-1$ nodes in $F_{\text {out }}\left(n_{i}\right)$. For the nodes in $F_{\text {out }}\left(n_{i}\right)$, we pick a node $n_{j}$ from $F_{\text {out }}\left(n_{i}\right)$ and remove eack node $n_{k} \in F_{\text {out }}\left(n_{j}\right)$ from $F_{\text {out }}\left(n_{i}\right)$. Since $n_{i}$ has at most $m-1$ fan-outs, and each of the fan-outs has at most $m-1$ fan-outs, we need $O\left(m^{2}\right)$ to find a reduction edge $\left(n_{i}, n_{j}\right)$.

\section{B. Solution Perturbation}

We apply the following four operations to perturb a TCG:

- Rotation: Rotate a module.

- Swap: Swap two nodes in both of $C_{h}$ and $C_{v}$.

- Reverse: Reverse a reduction edge in $C_{h}$ or $C_{v}$.

- Move: Move a reduction edge from one transitive closure graph $\left(C_{h}\right.$ or $\left.C_{v}\right)$ to the other.

Rotation and Swap do not change the topology of a TCG while Reverse and Move do. To maintain the properties of the TCG after performing the Reverse and Move operations, we may need to update the resulting graphs. We detail the four operations as follows.

\section{B.1 Rotation}

To rotate a module $b_{i}$, we only need to exchange the weights of the corresponding node $n_{i}$ in $C_{h}$ and $C_{v}$. Fig 6(b) shows the resulting $C_{h}, C_{v}$, and placement after rotating the module $d$ 


\begin{tabular}{|c|c|c|c|c|c|c|c|}
\hline Representation & SP [7] & "FAST-SP [16] & $\overline{\mathrm{BSG}[8]}$ & O-tree [2] & 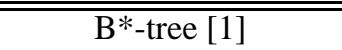 & CBL [3] & $\overline{\mathrm{TCG}}$ \\
\hline $\begin{array}{l}\text { Type of floorplans that } \\
\text { can be represented }\end{array}$ & " general & "general & 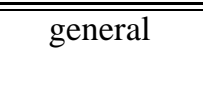 & \multicolumn{2}{|r|}{ compacted } & mosaic & $\overline{\text { general }}$ \\
\hline Is $\mathrm{P}^{*}$-admissible? & Yes & Yes & $\overline{Y e s}$ & \multirow{2}{*}{\multicolumn{2}{|c|}{$\frac{\text { No }}{O\left(m ! 2^{2 m} / m^{1.5}\right)}$}} & No & Yes \\
\hline Solution space & $(m !)^{2}$ & $(m !)^{2}$ & $m ! C\left(m^{2}, m\right)$ & & & $O\left(m ! 2^{3 m}\right)$ & $(m !)^{2}$ \\
\hline $\begin{array}{l}\text { Every solution } \\
\text { is feasible? }\end{array}$ & Yes & Yes & Yes & \multicolumn{2}{|c|}{ 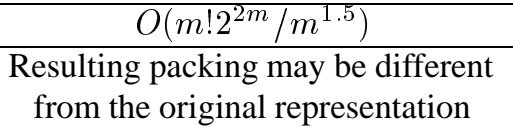 } & No & Yes \\
\hline Runtime for packing & $O\left(m^{2}\right)$ & $\begin{array}{c}\text { Amortized } \\
O(m \lg \lg m)\end{array}$ & $O\left(m^{2}\right)$ & \multicolumn{2}{|r|}{$O(m)$} & $O(m)$ & $O\left(m^{2}\right)$ \\
\hline $\begin{array}{l}\text { Best evaluated } \\
\text { packing corresp. to } \\
\text { optimal placement? }\end{array}$ & Yes & Yes & Yes & \multicolumn{2}{|c|}{$\begin{array}{l}\text { True for are optimization, } \\
\text { but not for wirelength } \\
\text { optimization }\end{array}$} & No & Yes \\
\hline $\begin{array}{l}\text { Geometric relation } \\
\text { between any two } \\
\text { modules is defined? }\end{array}$ & Yes & Yes & Yes & \multicolumn{2}{|r|}{ No } & No & Yes \\
\hline $\begin{array}{l}\text { Need module } \\
\text { sequence encoding? }\end{array}$ & Yes & Yes & No & Yes & No & Yes & No \\
\hline $\begin{array}{l}\text { Construct additional } \\
\text { constraint graphs } \\
\text { for packing? }\end{array}$ & Yes & No & Yes & Yes & No & Yes & No \\
\hline $\begin{array}{l}\text { Evaluate cost directly } \\
\text { on representation? }\end{array}$ & No & No & $\overline{\text { No }}$ & $\overline{\text { No }}$ & Yes & No & Yes \\
\hline $\begin{array}{l}\text { Geometric relation is } \\
\text { transparent to its } \\
\text { operations? }\end{array}$ & $\overline{\text { No }}$ & No & No & No & No & No & Yes \\
\hline $\begin{array}{l}\text { Boundary information } \\
\text { on representation? }\end{array}$ & No & No & $\overline{\text { No }}$ & $\overline{\text { No }}$ & $\overline{\text { No }}$ & $\overline{\text { No }}$ & Yes \\
\hline
\end{tabular}

TABLE I

Properties of RePresentATIONS. Here, $m$ IS THE NUMBER OF MODULES IN A PLACEMENT.

shown in Fig 6(a). Notice that the weights associated with the node $n_{d}$ in $C_{h}$ and $C_{v}$ have been exchanged.

Theorem 5: TCG is closed under the rotation operation, and such an operation takes $O(1)$ time.

Proof: We do not change a TCG for the Rotation operation, and thus the resulting graphs are still a TCG. It is obvious that exchanging the weights of a node in $C_{h}$ and $C_{v}$ takes $O(1)$ time.

\section{B.2 Swap}

To swap two nodes $n_{i}$ and $n_{j}$, we only need to exchange two nodes in both $C_{h}$ and $C_{v}$. Fig 6(c) shows the resulting $C_{h}, C_{v}$, and placement after swapping the nodes $n_{a}$ and $n_{b}$ shown in Fig 6(b). Notice that the nodes $n_{a}$ and $n_{b}$ in both $C_{h}$ and $C_{v}$ have been exchanged.

Theorem 6: TCG is closed under the swap operation, and such an operation takes $\mathrm{O}(1)$ time.

Proof: Since we only exchange two nodes in both $C_{h}$ and $C_{v}$ without changing the topology of a TCG for the Swap operation, the resulting graphs are still a TCG. Exchanging the corresponding pointers of two nodes in both $C_{h}$ and $C_{v}$ takes $O(1)$ time.

\section{B.3 Reverse}

The Reverse operation reverses the direction of a reduction edge $\left(n_{i}, n_{j}\right)$ in a transitive closure graph, which corresponds to changing the geometric relation of the two modules $b_{i}$ and $b_{j}$. For two modules $b_{i}$ and $b_{j}, b_{i} \vdash b_{j}\left(b_{i} \perp b_{j}\right)$ if there exists a reduction edge $\left(n_{i}, n_{j}\right)$ in $C_{h}\left(C_{v}\right)$; after reversing the edge $\left(n_{i}, n_{j}\right)$, we have the new geometric relation $b_{j} \vdash b_{i}\left(b_{j} \perp b_{i}\right)$. Therefore, the geometric relation among modules is transparent not only to the TCG representation but also to the Reverse operation (i.e., the effect of such an operation on the change of the geometric relation is known before packing); this property can facilitate the convergence to a desired solution.

To reverse a reduction edge $\left(n_{i}, n_{j}\right)$ in a transitive closure graph, we first delete the edge from the graph, and then add the edge $\left(n_{j}, n_{i}\right)$ to the graph. For each node $n_{k} \in F_{i n}\left(n_{j}\right) \cup$ $\left\{n_{j}\right\}$ and $n_{l} \in F_{\text {out }}\left(n_{i}\right) \cup\left\{n_{i}\right\}$ in the new graph, we shall check whether the edge $\left(n_{k}, n_{l}\right)$ exists in the new graph. If the graph contains the edge, we do nothing; otherwise, we need to add the edge to the graph and delete the corresponding edges $\left(n_{k}, n_{l}\right)$ (or $\left.\left(n_{l}, n_{k}\right)\right)$ in the other transitive closure graph, if any, to maintain the properties of the TCG.

Fig 6(d) shows the resulting $C_{h}, C_{v}$, and placement after reversing the reduction edge $\left(n_{c}, n_{e}\right)$ of the $C_{v}$ shown in Fig 6(c). In the $C_{v}$ of Fig 6(d), $F_{i n}\left(n_{e}\right) \cup\left\{n_{e}\right\}=\left\{n_{a}, n_{b}, n_{e}\right\}$ and 

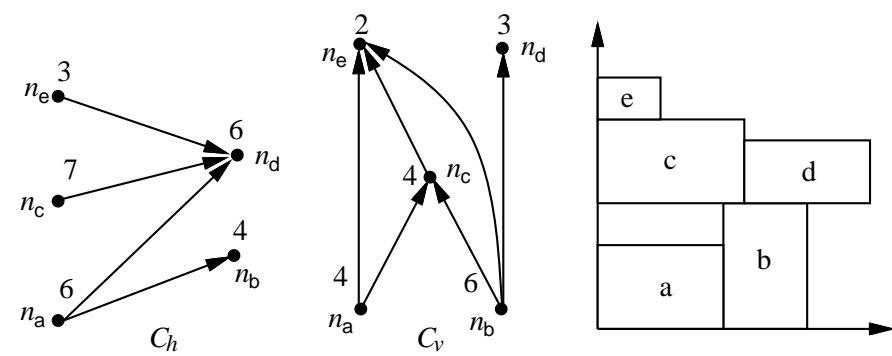

(a) initial configuration of TCGs
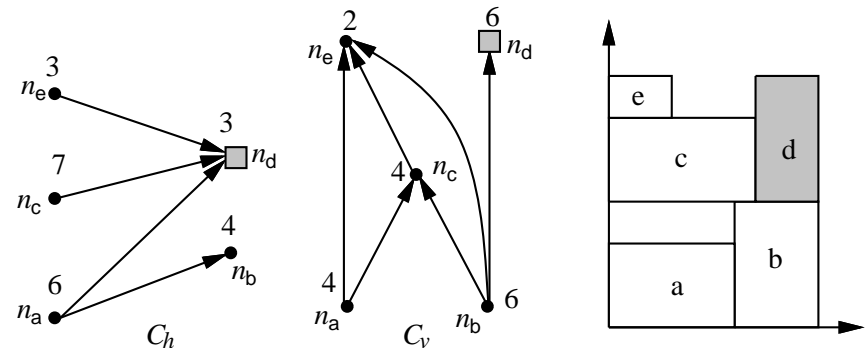

(b) rotate module $d$
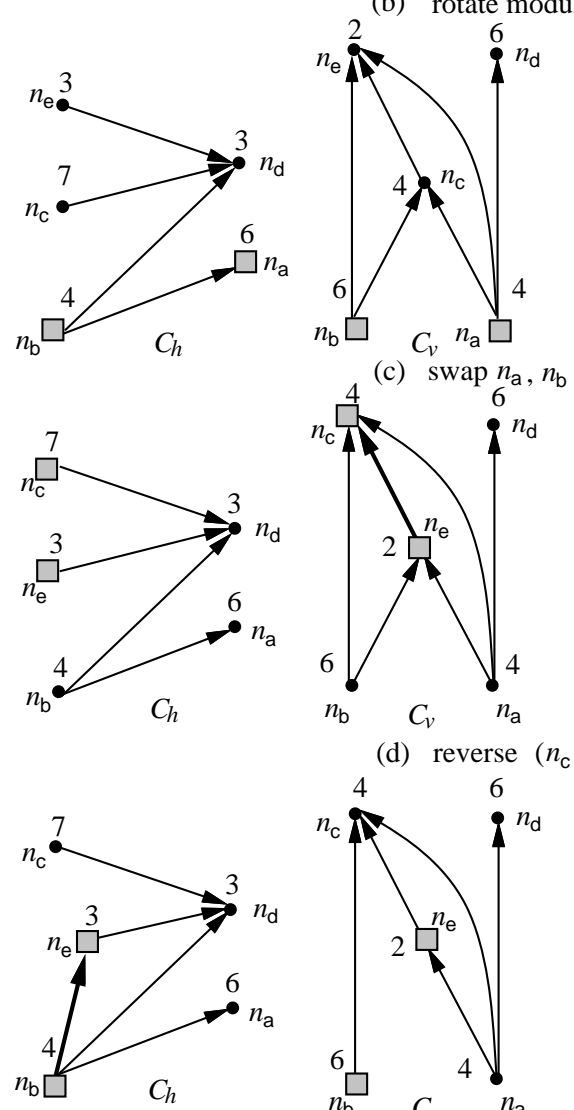

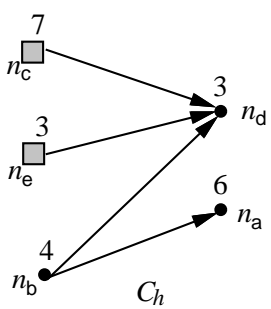

$C_{h}$ (c) swap $n_{\mathrm{a}}, n_{\mathrm{b}}$

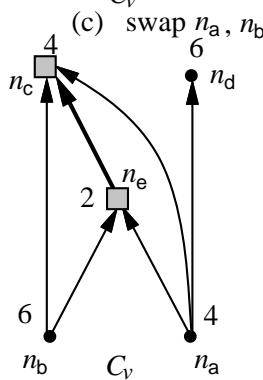

(d) reverse $\left(n_{\mathrm{c}}, n_{\mathrm{e}}\right)$

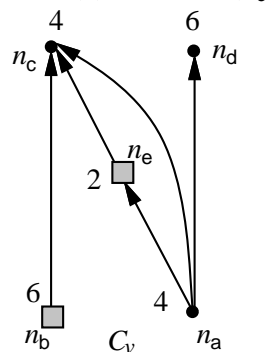

(e) move $\left(n_{\mathrm{b}}, n_{\mathrm{e}}\right)$
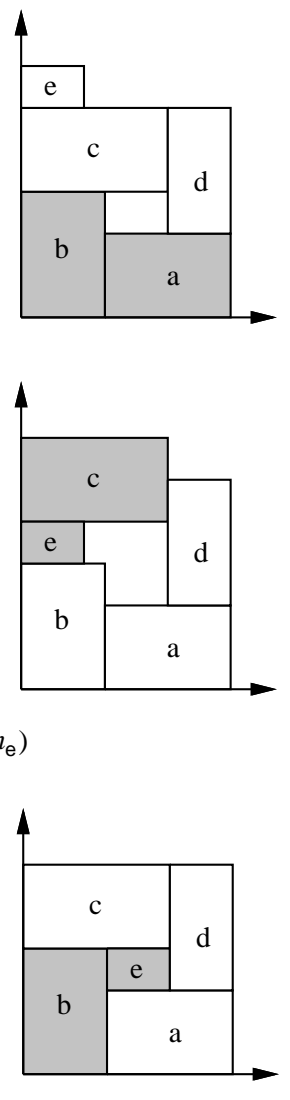

)

Fig. 6. Four types of perturbation. (a) The initial TCG $\left(C_{h}\right.$ and $\left.C_{v}\right)$ and placement. (b) The resulting TCG and placement after rotating the module $d$ shown in (a). (c) The resulting TCG and placement after swapping the nodes $n_{a}$ and $n_{b}$ shown in (b). (d) The resulting TCG and placement after reversing the reduction edge $\left(n_{c}, n_{e}\right)$ shown in (c). (e) The resulting TCG and placement after moving the reduction edge $\left(n_{b}, n_{e}\right)$ from the $C_{v}$ of (d) to $C_{h}$.
$F_{\text {out }}\left(n_{c}\right) \cup\left\{n_{c}\right\}=\left\{n_{c}\right\}$. For each node $n_{k} \in F_{\text {in }}\left(n_{e}\right) \cup\left\{n_{e}\right\}$ and $n_{l} \in F_{\text {out }}\left(n_{c}\right) \cup\left\{n_{c}\right\}$, we check whether the edge $\left(n_{k}, n_{l}\right)$ exists. Since the edge $\left(n_{e}, n_{c}\right)$ was just added to $C_{v}$ and the edges $\left(n_{b}, n_{c}\right)$ and $\left(n_{a}, n_{c}\right)$ already exist in $C_{v}$ of Fig 6(c), we do not need to add the three edges to $C_{v}$. Neither do we need to update the $C_{h}$ of Fig 6(c). Notice that by reversing the reduction edge $\left(n_{c}, n_{e}\right)$ in the $C_{v}$, we transform the relation $b_{c} \perp b_{e}$ into $b_{e} \perp b_{c}$ in the resulting placement of Fig 6(d).

To maintain the properties of a TCG, we can only reverse a reduction edge. For example, if we reverse a closure edge $\left(n_{i}\right.$, $\left.n_{k}\right)$ associated with the two reduction edges $\left(n_{i}, n_{j}\right)$ and $\left(n_{j}\right.$, $n_{k}$ ), a cycle $<n_{i}, n_{j}, n_{k}, n_{i}>$ is formed, and thus the resulting graphs are no longer a TCG. Further, for each edge introduced in a transitive closure graph, we remove its corresponding edge from the other graph. Therefore, there is always exactly one relation between each pair of modules.

Theorem 7: TCG is closed under the reverse operation, and such an operation takes $O\left(\mathrm{~m}^{2}\right)$ time, where $m$ is the number of modules in the placement.

Proof: We first show that the resulting graphs $C_{h}$ and $C_{v}$ of a TCG satisfy the three properties of TCG after performing the Reverse operation.

Without loss of generality, we focus on the case for reversing a reduction edge $\left(n_{i}, n_{j}\right)$ in $C_{h}$. For Property 1 , suppose that the new $C_{h}$ is not acyclic after the Reverse operation. Then, there must exist a path from $n_{i}$ to $n_{j}$ in $C_{h}$ before the operation, implying that $\left(n_{i}, n_{j}\right)$ is a closure edge, which is a contradiction. The new $C_{v}$ must also be acyclic since we do not add any edge into $C_{v}$ during the operation. For Property 2, each pair of nodes must be connected by exactly one edge either in the new $C_{h}$ or in the new $C_{v}$ after the operation because we delete the edge $\left(n_{i}, n_{j}\right)$ from $C_{v}$ after adding the edge into $C_{h}$. For Property 3 , suppose that the new $C_{h}$ is not a transitive closure of itself. Then, there exists a path $\left\langle n_{x}, \ldots, n_{j}, n_{i}, \ldots, n_{y}\right\rangle$ in the new $C_{h}$, but the $C_{h}$ does not contain the closure edge $\left(n_{x}, n_{y}\right)$. During the operation, for each node $n_{k} \in F_{i n}\left(n_{j}\right) \cup\left\{n_{j}\right\}$ and $n_{l} \in F_{\text {out }}\left(n_{i}\right) \cup\left\{n_{i}\right\}$ in $C_{h}$, we add the edges $\left(n_{k}, n_{l}\right)$ 's to the new $C_{h}$ and delete them from $C_{v}$. Therefore, at least one of the edges $\left(n_{x}, n_{j}\right)$ and $\left(n_{i}, n_{y}\right)$ does not exist in the original $C_{h}$; otherwise, we would have added the closure edge $\left(n_{x}, n_{y}\right)$ into the new $C_{h}$ during the Reverse operation. This implies that the original $C_{h}$ is not a transitive closure graph, contradicting to our assumption. It is clear that the deleted edges in $C_{v}$ are the closure edges of the new $C_{h}$, which cannot be the closure edges in $C_{v}$. Therefore, the new $C_{v}$ is still a transitive closure graph of itself.

The time complexity is dominated by checking whether the edges $\left(n_{k}, n_{l}\right)$ 's $\left(n_{k} \in F_{i n}\left(n_{j}\right) \cup\left\{n_{j}\right\}\right.$ and $n_{l} \in F_{\text {out }}\left(n_{i}\right) \cup$ $\left.\left\{n_{i}\right\}\right)$ exist in the new graph and by inserting and deleting the corresponding edges. Since there are at most $O(m) n_{k}$ 's and $O(m) n_{l}$ 's, the operation takes $O\left(m^{2}\right)$ time in total.

\section{B.4 Move}

The Move operation moves a reduction edge $\left(n_{i}, n_{j}\right)$ in a transitive closure graph to the other, which corresponds to switching the geometric relation of the two modules $b_{i}$ and $b_{j}$ between a horizontal relation and a vertical one. For two modules $b_{i}$ and $b_{j}, b_{i} \vdash b_{j}\left(b_{i} \perp b_{j}\right)$ if there exists a reduction edge 
$\left(n_{i}, n_{j}\right)$ in $C_{h}\left(C_{v}\right)$; after moving the edge $\left(n_{i}, n_{j}\right)$ to $C_{v}\left(C_{h}\right)$, we have the new geometric relation $b_{i} \perp b_{j}\left(b_{i} \vdash b_{j}\right)$. Therefore, the geometric relation among modules is also transparent to the Move operation.

To move a reduction edge $\left(n_{i}, n_{j}\right)$ from a transitive closure graph $G$ to the other $G^{\prime}$ in a TCG, we first delete the edge from $G$ and add it to $G^{\prime}$. Similar to the Reverse operation, for each node $n_{k} \in F_{\text {in }}\left(n_{i}\right) \cup\left\{n_{i}\right\}$ and $n_{l} \in F_{\text {out }}\left(n_{j}\right) \cup\left\{n_{j}\right\}$, we shall check whether the edge $\left(n_{k}, n_{l}\right)$ exists in $G^{\prime}$. If $G^{\prime}$ contains the edge, we do nothing; otherwise, we need to add the edge to $G^{\prime}$ and delete the corresponding edge $\left(n_{k}, n_{l}\right)$ (or $\left.\left(n_{l}, n_{k}\right)\right)$ in $G$, if any, to maintain the properties of the TCG.

Fig 6(e) shows the resulting $C_{h}, C_{v}$, and placement after moving the reduction edge $\left(n_{b}, n_{e}\right)$ in the $C_{v}$ of Fig 6(d) to $C_{h}$. In the $C_{h}$ shown in Fig 6(e), $F_{i n}\left(n_{b}\right) \cup\left\{n_{b}\right\}=\left\{n_{b}\right\}$ and $F_{\text {out }}\left(n_{e}\right) \cup\left\{n_{e}\right\}=\left\{n_{d}, n_{e}\right\}$. For each node $n_{k} \in F_{i n}\left(n_{b}\right) \cup$ $\left\{n_{b}\right\}$ and $n_{l} \in F_{\text {out }}\left(n_{c}\right) \cup\left\{n_{c}\right\}$, we check whether the edge $\left(n_{k}, n_{l}\right)$ exists in $G_{h}$. Since the edge $\left(n_{b}, n_{e}\right)$ was just added to $C_{h}$ and the edge $\left(n_{b}, n_{d}\right)$ already exists in $C_{h}$ of Fig $6(\mathrm{~d})$, we need to do nothing (except moving the reduction edge $\left(n_{b}, n_{e}\right)$ from $C_{v}$ to $C_{h}$ ). Neither do we need to update $C_{v}$ (except removing the edge $\left.\left(n_{b}, n_{e}\right)\right)$. Notice that by moving the reduction edge $\left(n_{b}, n_{e}\right)$ from $C_{v}$ to $C_{h}$, we transform the relation $b_{b} \perp b_{e}$ into $b_{b} \vdash b_{e}$ in the resulting placement shown in Fig 6(e).

To maintain the properties of a TCG, we can only move a reduction edge. If we move a closure edge $\left(n_{i}, n_{k}\right)$ associated with the two reduction edges $\left(n_{i}, n_{j}\right)$ and $\left(n_{j}, n_{k}\right)$ in one transitive closure graph to the other, then there exist a path from $n_{i}$ to $n_{k}$ in the two graphs, implying that $b_{i} \vdash b_{k}$ and $b_{i} \perp b_{k}$, which gives a redundant solution. Further, for each edge introduced in a transitive closure graph, we remove its corresponding edge from the other graph. Therefore, there is always exactly one relation between each pair of modules.

Theorem 8: TCG is closed under the move operation, and such an operation takes $O\left(\mathrm{~m}^{2}\right)$ time, where $m$ is the number of modules in the placement.

Proof: We first show that the resulting graphs $C_{h}$ and $C_{v}$ of a TCG satisfy the three properties of TCG after performing the Move operation.

Without loss of generality, we focus on the case for moving a reduction edge $\left(n_{i}, n_{j}\right)$ from $C_{h}$ to $C_{v}$. For Property 1 , suppose that the resulting $C_{v}$ is not acyclic after we move a reduction edge $\left(n_{i}, n_{j}\right)$ from $C_{h}$ to $C_{v}$. There must exist a path from $n_{j}$ to $n_{i}$ in the original $C_{v}$. This implies that the edge $\left(n_{j}, n_{i}\right)$ is also in the original $C_{v}$ since $C_{v}$ is a transitive closure graph. This is a contradiction since $\left(n_{i}, n_{j}\right)$ and $\left(n_{j}, n_{i}\right)$ cannot both exist in the original TCG (Property 2). Therefore, the new $C_{v}$ must be acyclic. The new $C_{h}$ must also be acyclic since we do not add any edge into the original $C_{h}$. For Property 2, each pair of nodes must be connected by exactly one edge either in the new $C_{h}$ or in the new $C_{v}$ after the operation because the corresponding edge will be deleted from $C_{h}$ after the edge $\left(n_{i}\right.$, $n_{j}$ ) is added to $C_{v}$. For Property 3 , suppose that the new $C_{v}$ is not a transitive closure of itself. Then, there exists a path $<n_{x}, \ldots, n_{i}, n_{j}, \ldots, n_{y}>$ in the new $C_{v}$, but the $C_{v}$ does not contain the closure edge $\left(n_{x}, n_{y}\right)$. During the operation, for each node $n_{k} \in F_{\text {in }}\left(n_{i}\right) \cup\left\{n_{i}\right\}$ and $n_{l} \in F_{\text {out }}\left(n_{j}\right) \cup\left\{n_{j}\right\}$ in $C_{v}$, we add the edges $\left(n_{k}, n_{l}\right)$ 's to the new $C_{v}$ and delete

\begin{tabular}{|c|c|c|c|c|}
\hline \hline Circuit & \#Modules & \#I/O pads & \#Nets & \#Pins \\
\hline apte & 9 & 73 & 97 & 214 \\
xerox & 10 & 107 & 203 & 696 \\
hp & 11 & 43 & 83 & 264 \\
ami33 & 33 & 42 & 123 & 480 \\
ami49 & 49 & 24 & 408 & 931 \\
\hline \hline
\end{tabular}

TABLE II

THE FIVE MCNC BENCHMARK CIRCUITS.

them from $C_{h}$. Therefore, at least one of the edges $\left(n_{x}, n_{i}\right)$ and $\left(n_{j}, n_{y}\right)$ does not exist in the original $C_{h}$; otherwise, we would have added the closure edge $\left(n_{x}, n_{y}\right)$ into the new $C_{v}$ during the Move operation. This implies that the original $C_{v}$ is not a transitive closure graph, contradicting to our assumption. It is clear that the deleted edges of $C_{h}$ are the closure edges of the new $C_{v}$, which cannot be the closure edges in $C_{h}$. Therefore, the new $C_{h}$ is still a transitive closure graph of itself.

Similar to the arguments in the proof of Theorem 7, the operation takes $O\left(\mathrm{~m}^{2}\right)$ time in total.

\section{EXPERIMENTAL RESULTS}

Based on a simulated annealing method [4], we implemented the TCG representation in the $\mathrm{C}++$ programming language on a $433 \mathrm{MHz}$ SUN Sparc Ultra-60 workstation with 1 GB memory. The TCG package is available at http://cc.ee.ntu.edu.tw/ ywchang/research.html. We compared TCG with O-tree [2], B*-tree [1], enhanced O-tree [13], and CBL [3] based on the five MCNC benchmark circuits listed in TABLE II. Columns 2, 3, 4, and 5 of TABLE II list the respective numbers of modules, I/O pads, nets, and pins of the five circuits.

The experiments consist of three parts: area optimization, wirelength optimization, and simultaneous area and wirelength optimization. The area of a placement is measured by that of the minimum bounding box enclosing the placement. The area and runtime comparisons among O-tree [2], $\mathrm{B}^{*}$-tree [1], enhanced O-tree [13], CBL [3], and TCG are listed in TABLE III. As shown in TABLE III, TCG achieves average improvements of $2.22 \%, 1.18 \%, 2.04 \%$, and $3.54 \%$ in area utilization compared to $\mathrm{O}$-tree, $\mathrm{B} *$-tree, enhanced $\mathrm{O}$-tree, and $\mathrm{CBL}$, respectively. The runtimes are significantly smaller than $\mathrm{O}$-tree and $\mathrm{B} *$-tree, and comparable to the enhanced O-tree [13]. Fig 7 (left) shows the resulting placement for ami49 with area optimization.

For wirelength optimization, we estimated the wirelength of a net by half the perimeter of the minimum bounding box enclosing the net. The wirelength of a placement is given by the summation of the wirelengths of all nets. The comparisons with the previous works are listed in TABLE IV. (Note that $\mathrm{B}^{*}$-tree and $\mathrm{CBL}$ did not report the results on optimizing wirelength alone.) As shown in TABLE IV, TCG achieves average reductions of $3.56 \%$ and $3.18 \%$ in wirelength, compared to the O-tree and the enhanced O-tree, respectively. Fig 7 (right) shows the resulting placement for ami49 with wirelength optimization. For simultaneous area and wirelength optimization, we assigned the same weight for area and wirelength in the cost function. The results 


\begin{tabular}{|c||c|c||c|c||c|c||c|c||c|c||c||c|}
\hline \hline \multirow{2}{*}{ Circuit } & \multicolumn{2}{c||}{ SP } & \multicolumn{2}{c||}{ O-tree } & \multicolumn{2}{c||}{ B*-tree } & \multicolumn{2}{c||}{ enhanced O-tree } & \multicolumn{2}{c|}{ CBL } & \multicolumn{2}{c|}{ TCG } \\
\cline { 2 - 12 } & $\begin{array}{c}\text { Area } \\
\left(\mathrm{mm}^{2}\right)\end{array}$ & $\begin{array}{c}\text { Time } \\
(\mathrm{sec})\end{array}$ & $\begin{array}{c}\text { Area } \\
\left(\mathrm{mm}^{2}\right)\end{array}$ & $\begin{array}{c}\text { Time } \\
(\mathrm{sec})\end{array}$ & $\begin{array}{c}\text { Area } \\
\left(\mathrm{mm}^{2}\right)\end{array}$ & $\begin{array}{c}\text { Time } \\
(\mathrm{sec})\end{array}$ & $\begin{array}{c}\text { Area } \\
\left(\mathrm{mm}^{2}\right)\end{array}$ & $\begin{array}{c}\text { Time } \\
(\mathrm{sec})\end{array}$ & $\begin{array}{c}\text { Area } \\
\left(\mathrm{mm}^{2}\right)\end{array}$ & $\begin{array}{c}\text { Time } \\
(\mathrm{sec})\end{array}$ & $\begin{array}{c}\text { Area } \\
\left(\mathrm{mm}^{2}\right)\end{array}$ & $\begin{array}{c}\text { Time } \\
(\mathrm{sec})\end{array}$ \\
\hline \hline apte & 48.12 & 13 & 47.1 & 38 & 46.92 & 7 & 46.92 & 11 & NA & NA & 46.92 & 1 \\
xerox & 20.69 & 15 & 20.1 & 118 & 19.83 & 25 & 20.21 & 38 & 20.96 & 30 & 19.83 & 18 \\
hp & 9.93 & 5 & 9.21 & 57 & 8.947 & 55 & 9.16 & 19 & - & - & 8.947 & 20 \\
ami33 & 1.22 & 676 & 1.25 & 1430 & 1.27 & 3417 & 1.24 & 118 & 1.20 & 36 & 1.20 & 306 \\
ami49 & 38.84 & 1580 & 37.6 & 7428 & 36.80 & 4752 & 37.73 & 406 & 38.58 & 65 & 36.77 & 434 \\
\hline \hline Comp. & $+5.04 \%$ & - & $+2.22 \%$ & - & $+1.18 \%$ & - & $+2.04 \%$ & - & $+3.54 \%$ & - & $0.00 \%$ & - \\
\hline
\end{tabular}

TABLE III

AREA AND RUNTIME COMPARISONS AMONG SP (ON SUN Ultra60), O-TREE (ON SUN UlTRA60), B*-TREE (ON SUn Ultra-I), ENHANCED O-TREE (on Sun Ultra60), CBL (on Sun Sparc 20), and TCG (on Sun Ultra60) For area optimization. (NA: Not Available.)

\begin{tabular}{|c||c|c||c|c||c|c|}
\hline \hline \multirow{2}{*}{ Circuit } & \multicolumn{2}{c||}{ O-tree } & \multicolumn{2}{c||}{ enhanced O-tree } & \multicolumn{2}{c|}{ TCG } \\
\cline { 2 - 7 } & $\begin{array}{c}\text { Wire } \\
(\mathrm{mm})\end{array}$ & $\begin{array}{c}\text { Time } \\
(\mathrm{sec})\end{array}$ & $\begin{array}{c}\text { Wire } \\
(\mathrm{mm})\end{array}$ & $\begin{array}{c}\text { Time } \\
(\mathrm{sec})\end{array}$ & $\begin{array}{c}\text { Wire } \\
(\mathrm{mm})\end{array}$ & $\begin{array}{c}\text { Time } \\
(\mathrm{sec})\end{array}$ \\
\hline \hline apte & 317 & 47 & 317 & 15 & 363 & 2 \\
xerox & 368 & 160 & 372 & 39 & 366 & 15 \\
hp & 153 & 90 & 150 & 19 & 143 & 10 \\
ami33 & 52 & 2251 & 52 & 177 & 44 & 52 \\
ami49 & 636 & 14112 & 629 & 688 & 604 & 767 \\
\hline \hline Comp. & $+3.56 \%$ & - & $+3.18 \%$ & - & $0.00 \%$ & - \\
\hline
\end{tabular}

TABLE IV

WIRELENGTH AND RUNTIME COMPARISONS AMONG O-TREE (ON SUN UltRA60), ENHANCED O-TREE (ON SUN UlTRA60), AND TCG (ON SUN ULTRA60) FOR WIRELENGTH OPTIMIZATION.

are listed in TABLE V, which shows that ours are slightly better than previous works. ${ }^{2}$
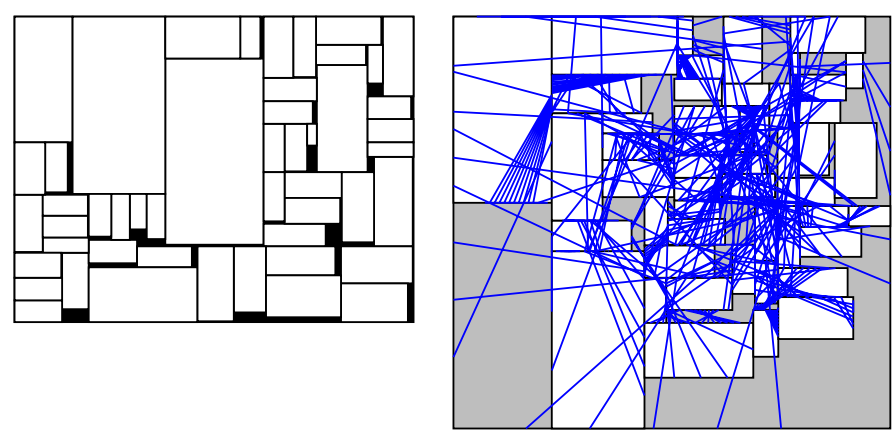

Fig. 7. Resulting placements of ami49 for (1) left: optimizing area alone (area $\left.=36.77 \mathrm{~mm}^{2}\right) ;(2)$ right: optimizing wirelngth alone (wire $=604 \mathrm{~mm}$ ).

Fig 8 show the stability and convergence-rate comparison between SP and TCG based on the circuit ami33. We randomly ran the programs for SP and TCG on ami33 ten times, based on the same initial solution each time. In Fig 8(a) and (b), the resulting areas are plotted as functions of the running times for SP and TCG using the same simulated annealing procedure. (Note that the parts with areas above $1.7 \mathrm{~mm}^{2}$ are not shown in the curves

\footnotetext{
${ }^{2}$ We excluded the CBL results for hp in TABLE III and for apte in TABLE V in the comparisons since the CBL test cases may not be the same as others. For example, CBL reports an area of $66.14 \mathrm{~mm}^{2}$ for hp, which is about seven times larger than others.
}

for clarity of the comparison.) As illustrated in Fig 8, TCG converges much faster to a desired solution and the results are much more stable than SP. For TCG, all of the ten runs converged in about $15 \mathrm{sec}$ and terminated in about $120 \mathrm{sec}$. We note that the stability and convergence rate should be very important metrics to evaluate the quality of a floorplan representation. However, they were often ignored in previous works.

\section{CONCLUDING REMARKS}

We have introduced the concept of the $\mathrm{P}^{*}$-admissible representation, presented the $\mathrm{P}^{*}$-admissible TCG representation for general floorplans, and shown its superior properties. Experimental results have shown that TCG is very efficient, effective, and stable in floorplan optimization. As revealed in the representation, TCG keeps the information of boundary modules as well as the shapes and the relative positions of modules. These properties make TCG a promising choice for dealing with the general floorplan/placement problems with various requirements. Research along this direction is ongoing.

\section{REFERENCES}

[1] Y.-C. Chang, Y.-W. Chang, G.-M. Wu, and S.-W. Wu, "B*-trees: A New Representation for Non-Slicing Floorplans," in Proc. DAC, 2000, pp. $458-463$

[2] P.-N. Guo, C.-K. Cheng, and T. Yoshimura, "An O-Tree Representation of Non-Slicing Floorplan and Its Applications," in Proc. DAC, 1999, pp. 268-273.

[3] X. Hong, G. Huang, Y. Cai, J. Gu, S. Dong, C.-K. Cheng, and J. Gu, "Corner Block List: An Effective and Efficient Topological Representation of Non-slicing Floorplan," in Proc. ICCAD, 2000, pp. 8-12.

[4] S. Kirkpatrick, C. D. Gelatt, and M. P. Vecchi, "Optimization by Simulated Annealing," Science, vol. 220, no. 4598, pp.671-680, May 13, 1983.

[5] E. Lawler, Combinatorial Optimization: Networks and Matroids, Holt, Rinehart, and Winston, 1976.

[6] J.-M. Lin and Y.-W. Chang, "TCG: A Transitive Closure Graph-Based Representation for Non-Slicing Floorplans," in Proc. DAC, 2001, pp. 764-769.

[7] H. Murata, K. Fujiyoshi, S. Nakatake, and Y. Kajitani, "RectanglePacking Based Module Placement," in Proc. ICCAD, 1995, pp. 472-479.

[8] S. Nakatake, K. Fujiyoshi, H. Murata, and Y. Kajitani, "Module Placement on BSG-Structure and IC Layout Applications," in Proc. ICCAD, 1996, pp. 484-491.

[9] Ohtsuki, T., N. Suzigama and H. Hawanishi, "An Optimization Technique for Intergrated Circuit Layout Design," in Proc. ICCST, 1970, pp. $67-68$.

[10] H. Onodera, Y. Taniquchi, and K. Tamaru, "Branch-and-bound Placement for Building Block Layout,” in Proc. DAC, 1991, pp. 433-439. 


\begin{tabular}{|c|c|c|c|c|c|c|c|c|c|c|c|c|}
\hline \multirow[b]{2}{*}{ Circuit } & \multicolumn{3}{|c|}{ O-tree } & \multicolumn{3}{|c|}{ enhanced O-tree } & \multicolumn{3}{|c|}{$\overline{\overline{C B L}}$} & \multicolumn{3}{|c|}{$\overline{\overline{\text { TCG }}}$} \\
\hline & $\begin{array}{c}\text { Area } \\
\left(\mathrm{mm}^{2}\right)\end{array}$ & $\begin{array}{l}\text { Wire } \\
(\mathrm{mm})\end{array}$ & $\begin{array}{l}\text { Time } \\
(\mathrm{sec})\end{array}$ & $\begin{array}{c}\text { Area } \\
\left(\mathrm{mm}^{2}\right)\end{array}$ & $\begin{array}{l}\text { Wire } \\
(\mathrm{mm})\end{array}$ & $\begin{array}{l}\text { Time } \\
(\mathrm{sec})\end{array}$ & $\begin{array}{c}\text { Area } \\
\left(\mathrm{mm}^{2}\right)\end{array}$ & $\begin{array}{l}\text { Wire } \\
(\mathrm{mm})\end{array}$ & $\begin{array}{l}\text { Time } \\
(\mathrm{sec})\end{array}$ & $\begin{array}{c}\text { Area } \\
\left(\mathrm{mm}^{2}\right)\end{array}$ & $\begin{array}{l}\text { Wire } \\
(\mathrm{mm})\end{array}$ & $\begin{array}{l}\text { Time } \\
(\mathrm{sec})\end{array}$ \\
\hline xerox & 20.42 & 380.6 & 142 & 20.42 & 380.6 & 41 & 20.233 & 403.47 & NA & 20.42 & 385.0 & 114 \\
\hline hp & 9.490 & 152.6 & 84 & 9.384 & 151.9 & 21 & NA & NA & NA & 9.490 & 151.8 & 59 \\
\hline ami33 & 1.283 & 51.31 & 2349 & 1.299 & 52.13 & 205 & 1.226 & 51.67 & NA & 1.237 & 50.29 & 939 \\
\hline Comp. & $+2.87 \%$ & $-2.01 \%$ & - & $+3.33 \%$ & $-1.34 \%$ & - & $-0.45 \%$ & $+5.98 \%$ & - & $0.00 \%$ & $0.00 \%$ & - \\
\hline
\end{tabular}

TABLE V

AREA, WIRELENGTH, AND RUNTIME COMPARISONS AMONG O-TREE (ON SUN UlTRA60), ENHANCED O-TREE (ON SUN ULTRA60), CBL (ON SUN SPARC 20), AND TCG (ON SUN ULTRA60) FOR SIMULTANEOUS AREA AND WIRELENGTH OPTIMIZATION.

[11] R. H. J. M. Otten, “Automatic Floorplan Design,” in Proc. DAC, 1982, pp.261-267.

[12] P. Pan and C.-L. Liu, "Area minimization for floorplans," IEEE Trans. on Computer-Aided Design, Vol. 14 no. 1, pp. 123-132, Jan. 1995.

[13] Y.-Pang, C.-K. Cheng, and T. Yoshimura, "An Enhanced Perturbing Algorithm for Floorplan Design using the O-tree Representation," in Proc. ISPD, 2000, pp. 168-173.

[14] S. M. Sait and H. Youssef, VLSI Physical Design Automation. McGrawHill, 1995.

[15] T. Takahashi, "A New Encoding Scheme for Rectangle Packing Problem," in Proc. ASP-DAC, 2000, pp. 175-178.

[16] X. Tang and D. F. Wong, "FAST-SP: A Fast Algorithm for Block Placement Based on Sequence Pair," in Proc. ASP-DAC, 2001, pp. 521-526.

[17] T.-C. Wang, and D. F. Wong, "An Optimal Algorithm for Floorplan and Area Optimization," in Proc DAC, 1990, pp.180-186.

[18] D. F. Wong, and C.-L. Liu, "A New Algorithm for Floorplan Design," in Proc DAC, 1986, pp. 101-107. 

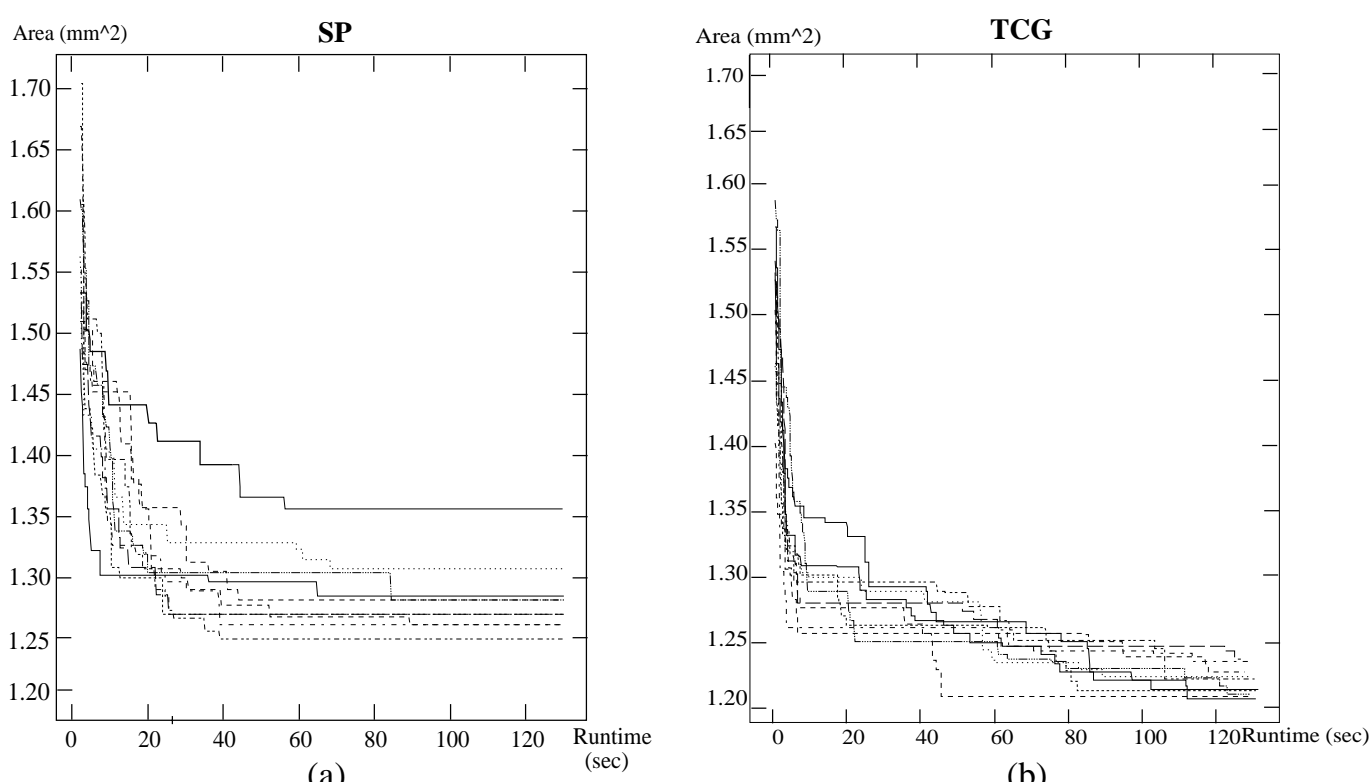

(a)

(b)

Fig. 8. Stability and convergence comparison between SP and TCG for ami33. (a) SP. (b) TCG. 